\title{
Effects of deposition dynamics on epitaxial growth
}

\author{
Jikeun Seo, ${ }^{1}$ Hye-Young Kim, ${ }^{2}$ and J.-S. Kim ${ }^{3}$ \\ ${ }^{1}$ Department of Ophthalmic Optics, Chodang University, Muan 534-701, Korea \\ ${ }^{2}$ Department of Physics, The Pennsylvania State University, University Park, PA 16802 \\ ${ }^{3}$ Department of Physics, Sook-Myung Women's University, Seoul, 140-742, Republic of Korea
}

(Dated: April 18, 2018)

\begin{abstract}
The dynamic effects, such as the steering and the screening effects during deposition, on an epitaxial growth $(\mathrm{Cu} / \mathrm{Cu}(001))$, is studied by kinetic Monte Carlo simulation that incorporates molecular dynamic simulation to rigorously take the interaction of the deposited atom with the substrate atoms into account. We find three characteristic features of the surface morphology developed by grazing angle deposition: (1) enhanced surface roughness, (2) asymmetric mound, and (3) asymmetric slopes of mound sides. Regarding their dependence on both deposition angle and substrate temperature, a reasonable agreement of the simulated results with the previous experimental ones is found. The characteristic growth features by grazing angle deposition are mainly caused by the inhomogeneous deposition flux due to the steering and screening effects, where the steering effects play the major role rather than the screening effects. Newly observed in the present simulation is that the side of mound in each direction is composed of various facets instead of all being in one selected mound angle even if the slope selection is attained, and that the slope selection does not necessarily mean the facet selection.
\end{abstract}

PACS numbers: PACS numbers:68.35.-p,68.37.-d

\section{INTRODUCTION}

For the tailored growth of a structure on a substrate, careful consideration of energetic parameters, such as surface and interface energies, and kinetic parameters, such as diffusion barriers, is necessary. However, the parameters related to the dynamics of the deposited atoms, other than the deposition flux, have not been seriously considered. Recently, Dijken, Jorritsma, and Poelsema 1 has observed by spot profile analysis low energy electron diffraction (SPA-LEED) that the islands formed on $\mathrm{Cu}$ (001) show rectangular symmetry in contrast to the square symmetry of the substrate when the 0.5 monolayer (ML) of $\mathrm{Cu}$ is deposited at deposition angle $(\theta)$ of $80^{\circ}$ from the surface normal. They suggest a model in which the interaction between deposited atom and substrate atoms steers the deposited atom and thus results in the inhomogeneous deposition flux, so called the steering effect. Our previous study on the thin film growth ${ }^{2}$, simulating dynamics of the deposited atoms by molecular dynamics (MD), has confirmed this hypothetical model. These studies demonstrate that the dynamic parameters involved in the deposition process, which have been ignored in most of the previous studies, exert notable effects on the thin film growth. Since the steering effect is unavoidable during deposition process, it influences all the thin film growth by atomic deposition in some degree. Even in the case of the normal deposition, the steering effect is found to affect the thin film growth $\frac{3.4 .5}{\text { It has }}$ been also shown that the deposition dynamics is a cause of the unstable growth of thin film on a vicinal surface ${ }^{6}$

Dijken, Jorritsma and Poelsema ${ }^{7}$ has observed, in the thicker film (40 ML) growth with $\theta=80^{\circ}$ that the surface is rougher than that with $\theta=0^{\circ}$. It has also been observed that the slope of the mound facing the depo- sition direction is much steeper than that of the side shadowed from the deposition $\underline{\underline{7}}$ Although a qualitative model is suggested relating the experimental result with the inhomogeneous deposition flux due to the steering and screening effects, $\stackrel{8}{=}$ there has not been any realistic growth study or simulation work which confirms such speculation.

In the present work, we perform kinetic Monte Carlo (KMC) simulation in conjunction with MD simulation that is designed to probe the effects of the dynamic processes on the thick film growth in atomic level. The main results of our simulation are as follows; (1) the roughness increases with increasing deposition angle, (2) the mound formed in the thick film growth has rectangular symmetry with sides elongated in the direction perpendicular to the deposition direction, and (3) the slopes of the illuminated and shadowed sides of the mound significantly differ, which is consistent with the experimental results? The aforementioned three characteristic morphological features are mainly caused by the inhomogeneous deposition flux on the top terrace of the mound mainly due to the steering effects rather than the screening effects. In the present study, in addition, it is found that the side of mound in each direction is formed of various local facets instead of all being in one selected mound angle, even if the slope selection is attained, and that the experimentally observed mound slope actually corresponds to the mean slope of various local facets coexisting on each mound side. Also found is that the dependence of the mound slope on growth condition is due to the variation of the relative population of the facets. 
TABLE I: diffusion barriers and parameters adopted in our simulation

\begin{tabular}{cc}
\hline \hline type of diffusion & diffusion barrier \\
\hline single adatom hopping(E1) & $0.48 \mathrm{eV}$ \\
step edge diffusion (E2) & $0.44 \mathrm{eV}$ \\
dimer lateral bond breaking (E3) & $0.46 \mathrm{eV}$ \\
re-estbilishing of a NN bond (E6) & $0.18 \mathrm{eV}$ \\
ES barrier (ES) & $0.10 \mathrm{eV}$ \\
ES barrier (kink site) & $0.05 \mathrm{eV}$ \\
\hline jump frequency $\left(\nu_{0}\right)$ & $2.4 \times 10^{13}$ \\
deposition rate $\left(F_{0}\right)$ & $0.00416 \mathrm{ML} / \mathrm{s}$ \\
\hline \hline
\end{tabular}

\section{SIMULATION SCHEMES}

Kinetic Monte Carlo (KMC) simulation is utilized to study the thin film growth by deposition. In most of the previous KMC simulations, the deposition process is treated by randomly or uniformly positioning atoms at arbitrary adsorption sites. In the present study, when the deposition process is selected during usual KMC routine, an MD routine is called to simulate the trajectory of a deposited atom by fully considering the interaction between the deposited atom and substrate atoms.

The details of the simulation are as follows. The substrate, $\mathrm{Cu}(001)$, lies on the $\mathrm{xy}$-plane $($ at $\mathrm{z}=0$ ) with $\mathrm{x}$ axis lying parallel to the [110] direction. In the deposition process, the deposition starts at the height of 11-28 $a_{z}$ above the substrate, and deposited atoms are incident at an angle in the direction of x-axis. Here, $a_{z}$ is the interlayer spacing of $\mathrm{Cu}(001)$. The interaction between a deposited atom and substrate atoms is calculated by summing pairwise Lennard-Jones potentials, $U(r)=4 D\left[(\sigma / r)^{12}-(\sigma / r)^{6}\right]$. Here, $D=0.4093 \mathrm{eV}$, $\sigma=2.338 \AA$,, 9 and $r$ is the distance between two atoms. The initial kinetic energy of the deposited atom is set to $0.15 \mathrm{eV}$, which corresponds to the melting temperature of copper. During each deposition process, all the substrate atoms are assumed to be frozen in their positions. Verlet algorithm is used in the MD simulation.

In between two sequential deposition processes, KMC simulation is performed to simulate the diffusion processes of atoms on the substrate. In the KMC, only the diffusion into empty lattice sites is allowed and the exchange diffusion is not allowed. Also the overhang is not allowed during both deposition and diffusion processes. The simulation system is composed of $400 \times 400$ atomic lattice sites in fcc (001) surface and a vacuum region on top of the substrate with height of 28 atomic layers.

Values of diffusion coefficients and diffusion barriers are adopted from those used by Furman and coworkers, 11.12 which reproduced the surface morphology of $\mathrm{Cu}$ islands on $\mathrm{Cu}(001)$ very well; the step EhrlichSchwoebel (ES) barrier is $0.10 \mathrm{eV}$ and the kink ES barrier is $0.05 \mathrm{eV}$. In total, eleven kinds of diffusion barriers (including the ES barriers) are used in the KMC simulation and some of the important diffusion barriers are listed

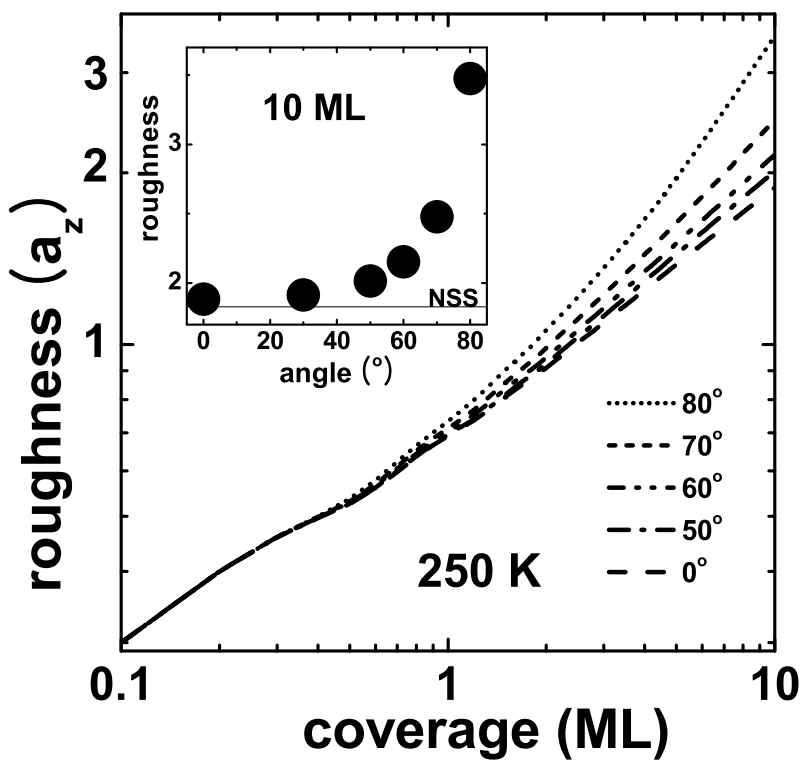

FIG. 1: Surface roughness as a function of coverage at $250 \mathrm{~K}$. Inset shows the roughness as function of the deposition angle at $10 \mathrm{ML}$ coverage. The solid line in the inset is the simulation result of random deposition without considering the steering or screening effect (NSS: No Steering or Screening effect).

in Table I. Note that the barrier (E2) for the diffusion along step edge is $0.44 \mathrm{eV}$ in the present study, which is much larger than the generally accepted values of 0.2 $\sim 0.3 \mathrm{eV}$, to save the computation time for the very frequent diffusions back and forth along steps. We examine the dependence of the surface morphology on E2, and find that that the morphology does not show any noticeable dependence on $\mathrm{E} 2$ down to $0.34 \mathrm{eV}$, the lowest tested value of E2. In addition, the simulation with the E2 fairly reproduces the real growth mode. Hence, we anticipate that the high E2 value would not seriously limit the validity of the present simulation.

The surface roughness is determined by the root-meansquare fluctuation of surface height around the mean height. The mound radius is determined as the radius (r) that makes the first zero of the height-height correlation function $G(\vec{r})=<h(\vec{r}) h(0)>-<h(0)>^{2}$, and the mound radii along $\mathrm{x}$ - and $\mathrm{y}$-axis are calculated separately. All the results presented are obtained from the average of 20 simulations under identical conditions. Unless mentioned otherwise, the distance in a plane is in unit of $\mathrm{a}_{n n}$, and that in the vertical direction is in unit of $\mathrm{a}_{z}$. Here, the nearest neighbor distance, $\mathrm{a}_{n n}$, is $a_{n n}=a / \sqrt{2}$, and the interlayer distance is $a_{z}=a / 2$, where $a$ is the lattice constant of $\mathrm{Cu}$. 


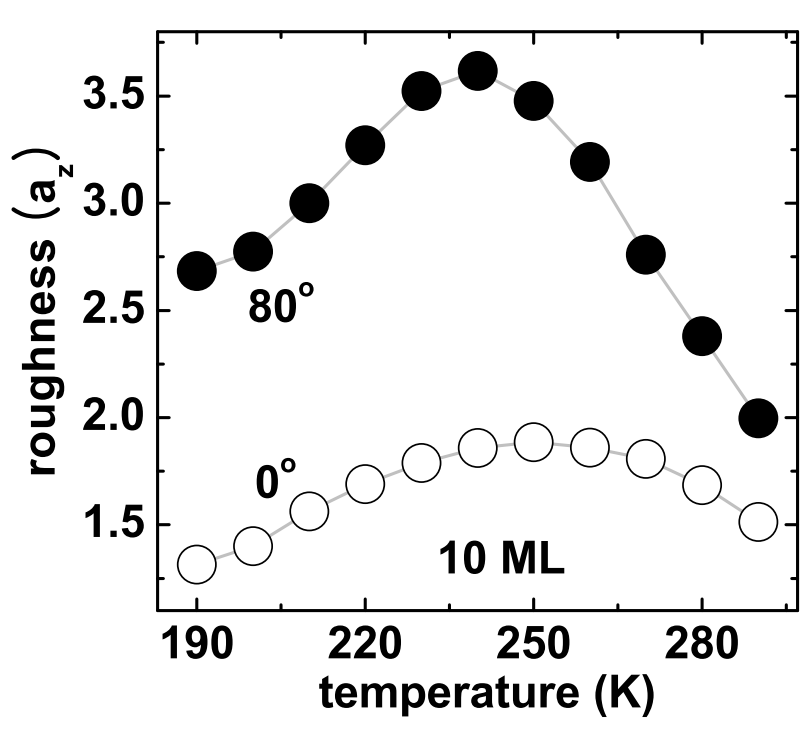

FIG. 2: Surface roughness as a function of temperature at coverage of $10 \mathrm{ML}$ for two deposition angles of $80^{\circ}$ and $0^{\circ}$ (normal incidence). The temperature dependence is more distinct for $80^{\circ}$ case than $0^{\circ}$ case.

\section{RESULTS}

\section{A. Roughness}

In Fig. 1, the surface roughness is presented as a function of the coverage $(\Theta)$ when $\mathrm{Cu}$ atoms are deposited at various deposition angles on $\mathrm{Cu}(001)$ at $250 \mathrm{~K}$. The most notable feature is that, even at the same coverage, the surface becomes much rougher as the deposition angle increases. Also, as $\Theta$ increases, the difference in roughness between the grazing angle deposition and the normal deposition $\left(\theta=0^{\circ}\right)$ multiplies. At $\Theta=10 \mathrm{ML}$, the roughness with $\theta=80^{\circ}$, comes to be twice larger than that with the normal deposition. (Inset of Fig. 1) The presently observed dependence of the roughness on the deposition angle is expected to originate from the deposition dynamics rather than the diffusion kinetics of the atoms on the substrate on the following grounds; (1) such angle dependence of the roughness is observed irrespective of the substrate temperature (T) (Fig. 2), and (2) both steering and screening effects become more effective as $\theta$ increases, as revealed by the simulation.(Fig.1 Inset)

In Fig. 2, shown is the dependence of the roughness on $T$; the roughness tends to decrease when $T$ becomes too high or too low. The bell - shape curve is wellexplained by the $T$-dependence of the destabilizing current by the ES barrier 13 Thus, such $T$-dependence is caused by the kinetics of deposited atoms and is irrelevant to the deposition dynamics. When the deposition is made at $\theta=80^{\circ}$, such bell-shape $T$-dependence is also observed, but now in an amplified form. This illustrates that the surface roughness is determined synergetically by the diffusion kinetics and the deposition dynamics.

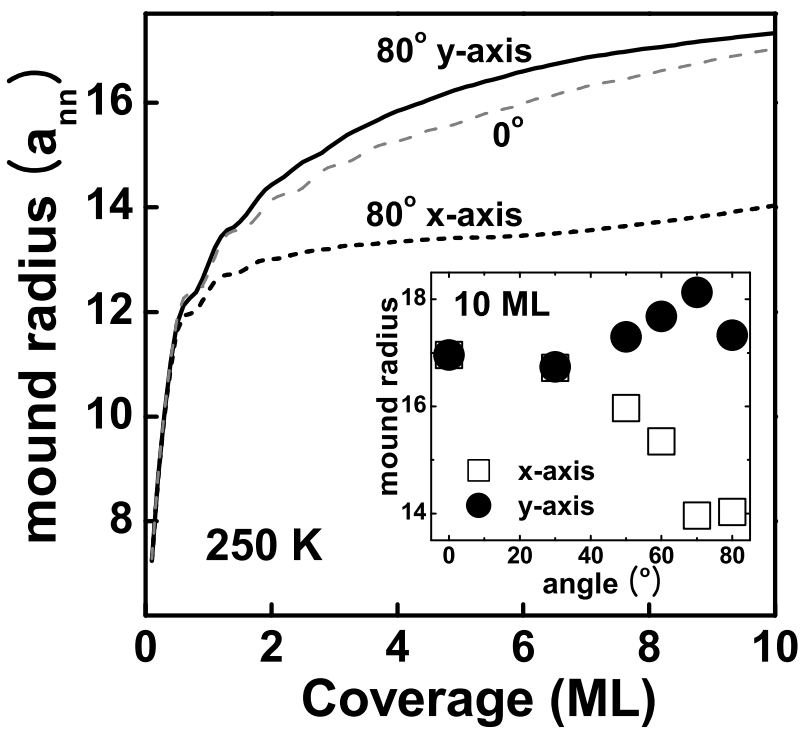

FIG. 3: Mound radius as a function of coverage. Results for $\theta=80^{\circ}$ are shown in solid and broken curve for radius along $\mathrm{x}$ - and $\mathrm{y}$-axis, respectively. Result for the normal deposition $\left(\theta=0^{\circ}\right)$ is also shown in gray broken curve. The substrate temperature is $250 \mathrm{~K}$ for all the cases. Inset: the mound radius as a function of the deposition angle at the coverage of $10 \mathrm{ML}$. Open squares and closed circles signify the radii along $\mathrm{x}$ - and $\mathrm{y}$-axis, respectively.

\section{B. Mound radius}

The mound radius as a function of the coverage and the deposition angle is shown in Fig. 3. When atoms are deposited in normal direction $\left(\theta=0^{\circ}\right)$, square mounds form with the same four-fold symmetry as that of the substrate. However, when the atoms are deposited at grazing angles, rectangular mounds form with evidently elongated sides along $\mathrm{y}$-axis. (Here, $\mathrm{x}(\mathrm{y})$-axis is parallel (perpendicular) to the deposition direction.) It is conspicuous, from Fig. 3 and its inset, that the difference between mound radii in $\mathrm{x}$ - and $\mathrm{y}$-axis increases as does the coverage or the deposition angle. This prediction agrees well with the experimental results of Dijken et $a l^{\underline{\underline{7}}}$ and $\mathrm{Lu}$ et $a l \stackrel{14}{\underline{14}}$, where such side way growth of the mound with high aspect ratio has been observed with the deposition at grazing angle. In regards to Fig. 3, the anisotropy in the shape of mound is caused mainly because the growth of the mound along $\mathrm{x}$-axis slows down once it reaches a certain length, $\simeq 13 a_{n n}$.

Fig. 4 shows $T$-dependence of the mound radii in two directions when $10 \mathrm{ML}$ film is grown at $\theta=80^{\circ}$. It shows that both mound radii increase as does $T$ due to the increased atomic diffusion length. At both high and low $T$ regimes, the mound shape is very asymmetric. The inset of Fig. 4 shows the aspect ratio as a function of $T$ at $\Theta=$ $10 \mathrm{ML}$, and the difference between the two mound radii as high as $40 \%$. (Here, the aspect ratio is defined as the ratio of the mound radius along $y$-axis to that along $\mathrm{x}$-axis.) The complicated dependence of the aspect ratio 


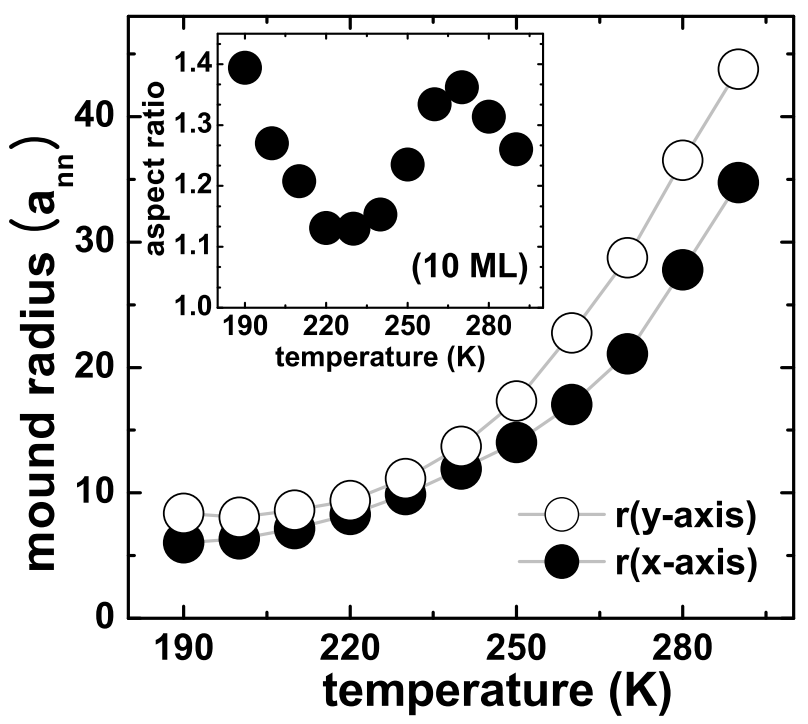

FIG. 4: Mound radius as a function of temperature for the deposition angle of $80^{\circ}$ and the coverage of $10 \mathrm{ML}$. Open and closed circles are for the radius along $\mathrm{x}$ - and $\mathrm{y}$-axis, respectively. Inset: the aspect ratio of the mound radii as a function of temperature.

on the temperature suggests that the diffusion kinetics also plays a substantial role in the determination of the mound shape in conjunction with the deposition dynamics. The asymmetric mound shape is, however, found over the whole $T$-range, which illustrates that the effect of deposition dynamics on the mound shape is never wiped out by the diffusion kinetics over the examined $T$-range.

\section{Mound slope}

For the characterization of the slopes of the mound, we investigate the local slope at each step on each side of the mound that is defined as the step height divided by the width of the adjacent lower terrace. Most steps in the sides of the mounds are of one-atomic-layer height. Thus, if the width of a lower terrace adjacent to a step is $0.5 \mathrm{a}_{n n}$, then, the local slope is $\mathrm{a}_{z} / 0.5 \mathrm{a}_{n n}$ which corresponds to the slope of $\{1,1,1\}$-facet on the fcc (001) surface. For the steps with the terrace widths, 1.5 and 2.5 $a_{n n}$, the corresponding local slopes are those of $\{1,1,3\}$ and $\{1,1,5\}$-facets, respectively.

In our simulation, we find that various kinds of steps coexist on each side of mound. In Fig. 5, the distribution of the various steps with different local slopes is presented as a function of the coverage for the thin films grown by the deposition at $\theta=0^{\circ}$ and $T=250 \mathrm{~K}$. As $\Theta$ increases, so does the mean slope. (Inset of Fig. 5) At $\Theta=100 \mathrm{ML}$, the mean terrace width becomes 1.67 $a_{n n}$ that is close to that of the $\{1,1,3\}$-facet. However, at the coverage, the relative population of the step with the local slope of $\{1,1, j\}$-facet (from now on, referred as $\{1,1, \mathrm{j}\}$-step $)$ is $21 \%(\mathrm{j}=1), 53 \%(\mathrm{j}=3), 18 \%(\mathrm{j}=5), 6$

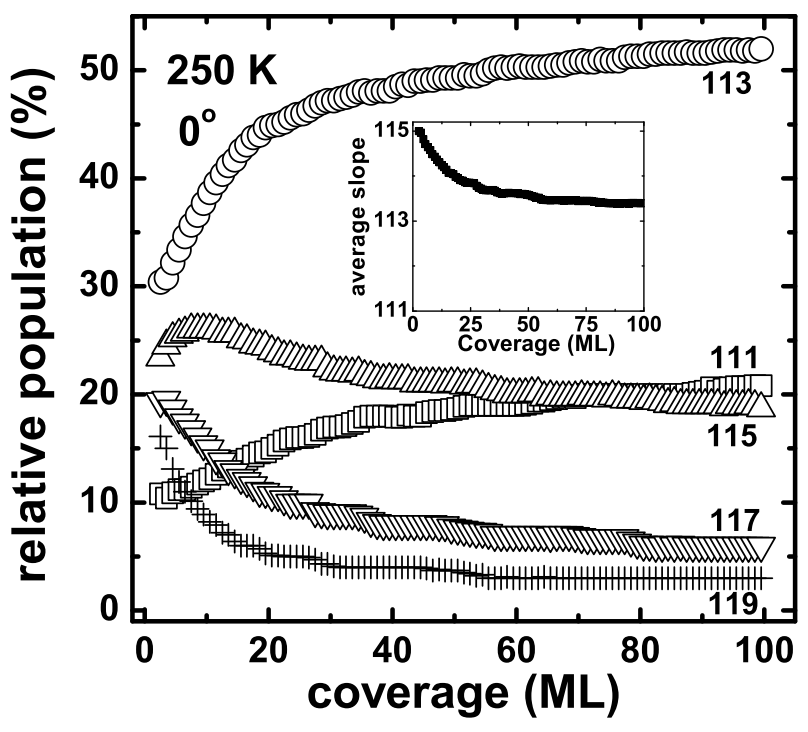

FIG. 5: Distribution of the facets that compose the sides of the mounds formed with the normal deposition $\left(0^{\circ}\right)$ at $250 \mathrm{~K}$. Inset: the average slope of the mounds as a function of the coverage.

$\%(\mathrm{j}=7)$, and $2 \%(\mathrm{j}=9)$. (The portion of steps with their slopes less than that of $\{1,1,11\}$-facet is negligible.) Therefore, even though the mean slope converges to that of $\{1,1,3\}$-facet, the actual relative population of steps with the mean slope is only $50 \%$ and the rest of the mounds are composed of the steps with relatively wide range of the local slopes.

Now, we examine the dependence of the distribution of slopes or steps on the deposition angle for three inequivalent sides of the mounds, illuminated (IL), shadowed $(\mathrm{SH})$, and perpendicular $(\mathrm{P})$ sides. (See the caption of Fig. 6 for the description of the sides.) In Fig. 6 shown is the distribution of local steps on each side after depositing $10 \mathrm{ML}$ at $250 \mathrm{~K}$ at various deposition angles. From the figure, we find that the distributions of the steps are similar for these three sides of the mounds for $\theta<50^{\circ}$. As the deposition angle further increases, the distributions start to change and become quite distinct from each other at $\theta=80^{\circ}$. For $\Theta=10 \mathrm{ML}$ and $\theta=80^{\circ}$, $54 \%$ of the IL-sides are composed of $\{1,1,1\}$-step. On the other hand, only $30 \%$ of SH-side is composed of the $\{1,1,1\}$-step, and thus its mean slope is much less steep than that of the IL-side. In the SPA-LEED experiment of $\mathrm{Cu} / \mathrm{Cu}(001)$, Dijken et al $\underline{\text { ? }}^{7}$ reported that the IL-side is made of $\{1,1,1\}$-step and SH- and P-sides are of $\{1,1,3\}$-step. These experimental results nearly match the average behaviors observed in our simulation.

We also investigate the distribution of the steps as a function of the substrate temperature (Fig. 7). For the case of grazing angle deposition, the step distributions (Fig. 7 (b)-(d)) and the mean slopes(Fig. 7 (e)) on the three sides become similar around $220 \mathrm{~K}$. Further, they vary little, as the substrate temperature is lowered below $220 \mathrm{~K}$. These observations suggest that the step distribu- 


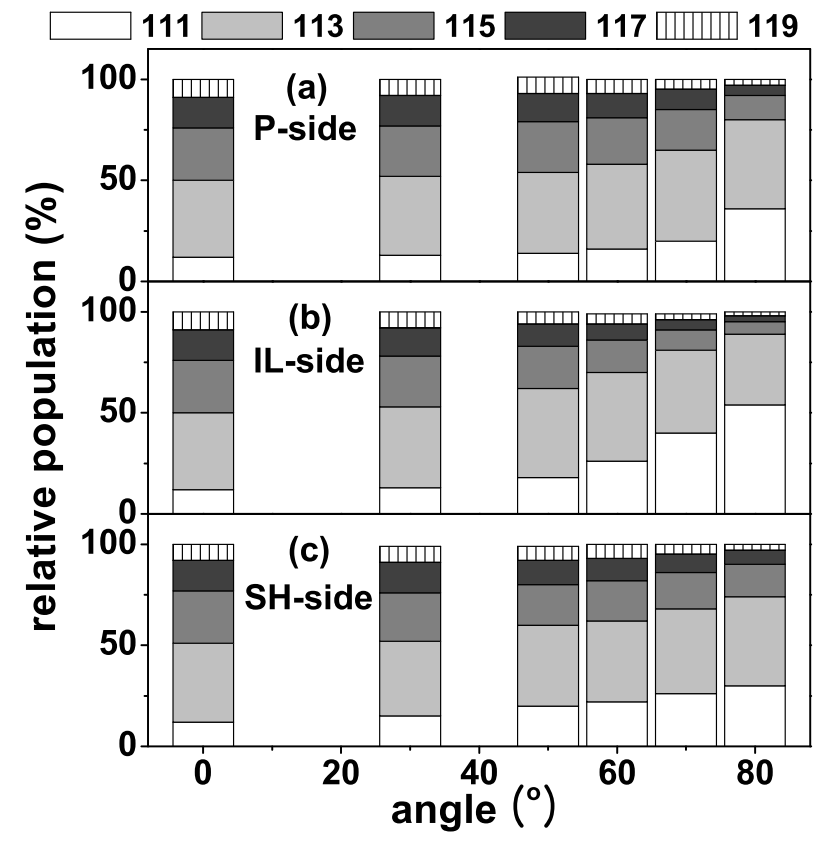

FIG. 6: Distribution of the facets that compose the sides of the mounds formed after depositing $10 \mathrm{ML}$ at $250 \mathrm{~K}$ at various deposition angles. (a) P-side: Two sides of mound facing in the direction perpendicular to the deposition direction, (b) IL-side: the side facing the deposition direction, and (c) SHside: the shadowed (or back) side of mound in the deposition direction.

tion for $T<220 K$ represents the limiting behavior of the slope formation or the steepest slope reachable by deposition, during which some less steep steps such as $\{113\}$-steps other than the steepest $\{111\}$-steps occurs due to statistical fluctuation.

As $T$ increases, the portion of the less steep steps increases and that of the steeper steps such as $\{111\}$-step decreases. That is, the mound becomes smooth as $T$ increases. The way of smoothing, however, differs depending on the mound side: As $T$ increases, the P-side (Fig. 7(b)) and the SH-side (Fig. 7(d)) rapidly become smooth, while the change in the step distribution on the IL-side occurs in the smaller scale (Fig. 7(c)). This can also be seen clearly in the $T$ dependence of the mean slopes in Fig. 7(e).

\section{DISCUSSION}

A. Effects of the deposition dynamics on the morphology of the film

In the previous section, we observe that the roughness (Inset of Fig. 1 and 2), the mound shape (Fig. 3 and 4) and the mound slope (Fig. 5-7) depend on the non-kinetic variable such as deposition angle. Such angular dependence can be attributed to the deposition dynamics that includes (1) the steering of the trajectory of
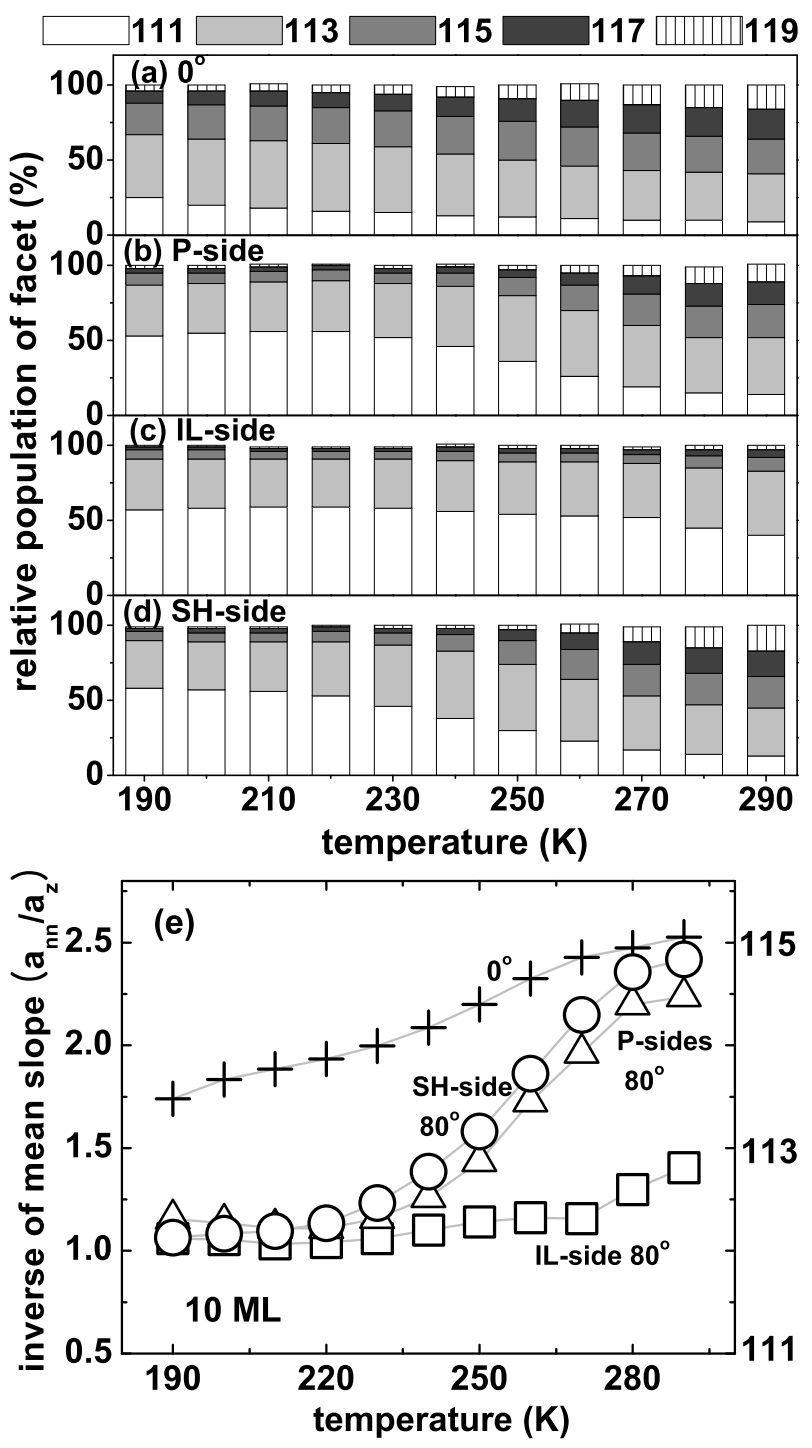

FIG. 7: Relative population of various facets and average facet slope at the coverage of $10 \mathrm{ML}$ as a function of temperature for both deposition angles of (a) $0^{\circ}$ and (b-d) $80^{\circ}$. (e)Mean slopes of the three sides of the mound as a function of the deposition temperature for the coverage of $10 \mathrm{ML}$.

the deposited atom due to its interaction with substrate atoms and (2) the screening effects of the deposited atoms due to the geometrical structure already formed on the substrate. Both effects cause inhomogeneous deposition flux depending on the deposition condition and make the growth of thin films sensitive to the deposition condition.

To study the effects of the inhomogeneous deposition flux on the growth of thin film, we investigate the flux on an 8-layer high mound surrounded with $\{1,1,5\}$-facets by MD simulation. Fig. 8(a) shows the deposition flux over the mound in gray scale for $\theta=80^{\circ}$. Fig. $8(\mathrm{~b})$ shows the deposition flux along a line passing through the center of the mound along the $\mathrm{x}$-axis, which shows strong asymmetry, i.e., the deposition flux on the IL-side is 2 to 4 times larger than the average deposition flux and that 


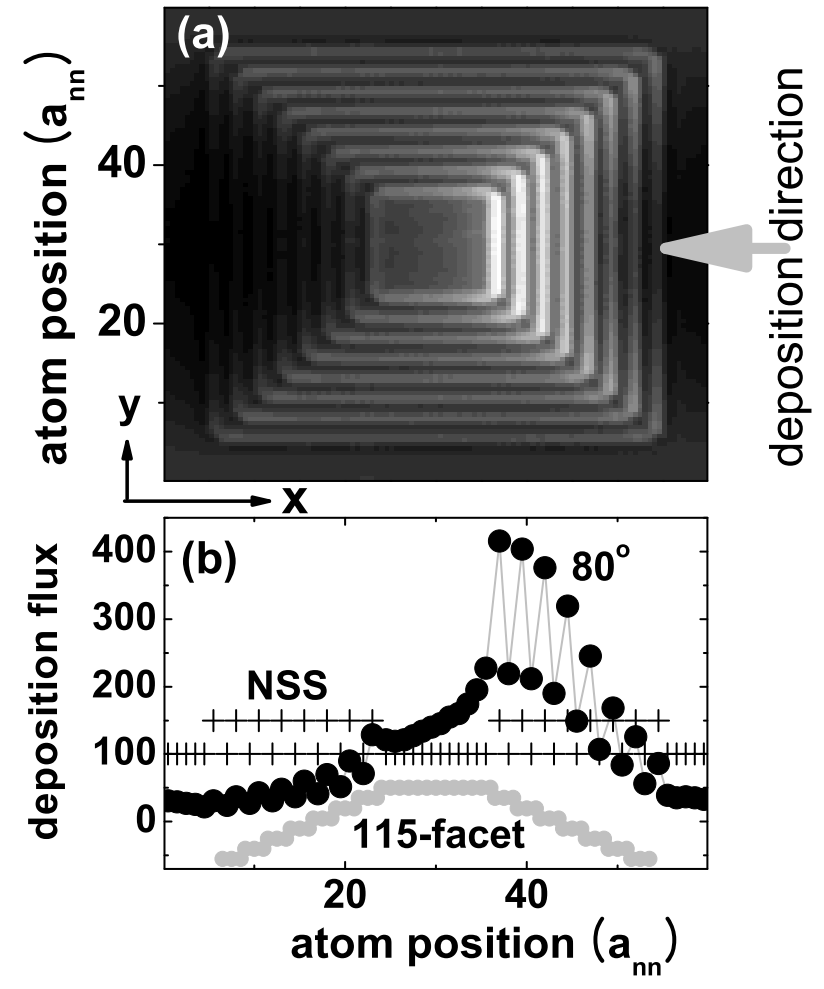

FIG. 8: Deposition flux calculated by MD simulation. Atoms are deposited at grazing deposition angle of $80^{\circ}$ on an 8-layer high mound surrounded by $(1,1,5)$-facets. (a) The local deposition flux in gray scale, where the brighter color indicates the higher local flux. (b) The local deposition flux along the line crossing the center of the mound along x-axis. The ordinate is the deposition flux in percentage relative to the average deposition flux over the total area. The deposition flux at normal $\left(0^{\circ}\right)$ deposition is shown with + symbol as a reference and the mound is depicted by gray circles at the bottom.

on SH-side is only about 10 to $50 \%$ of the average. The simulation with the mounds surrounded by the different facets show the same trend, i.e., the enhanced deposition flux on the IL-side and the reduced deposition flux on the SH-side.

Such inhomogeneous deposition flux gives rise to different growth speeds on each side, and thus the film is rougher than the film grown under the normal deposition condition (Figs.1 and 2). Especially, the enhanced deposition flux near the front edge of the top terrace increases the destabilizing current toward ascending step edge by the ES barrier, and critically roughens the surface ${ }^{13}$ (See the following section for the detailed description.)

The asymmetric shape of mound formed during grazing angle deposition (Fig. 3 and 4 ) is attributed to the decrease of the overall deposition flux along x-direction (i.e., over both the IL- and SH-sides), and the simultaneous increase of the effective deposition flux over the P-sides (Fig. 8) due to the following two reasons; the first one is the net mass transfer from the region near the rear edge of the mound to the top of the mound due to the steering effects, reducing the deposition flux along $\mathrm{x}$-axis $\stackrel{1.2}{=}$ Some of the transferred mass on the top of the mound is in the long run redistributed equally to the four sides ${ }_{2}^{2}$ It effectively increases the deposition flux along the $\mathrm{y}$-axis and vice versa. The second one is the increased deposition flux over the P-sides due to the attraction of the deposited atoms moving along the edges of the P-sides toward the sides ${ }^{2}$ Such imbalance of the effective deposition flux results in the faster growth speed in the y-direction than in the $\mathrm{x}$-direction, giving birth to the asymmetric mounds elongated along y-axis.

The observed asymmetric slopes of the mounds observed for $\theta>50^{\circ}$ (Fig. 6) can also be explained by the inhomogeneous deposition flux on the following two grounds. Firstly, the higher deposition flux on IL-side than that on SH-side offers growth environment effectively equivalent to the lower growth temperature on ILside than that on SH-side. Thus, the terrace size in ILside is narrower than that in SH-side or, equivalently, the slope in IL-side is steeper than that in SH-side as observed in both the previous experiment ${ }^{\underline{7}}$ and the present simulation.

In addition, the flux distribution on the top terrace strengthens the asymmetric slope formation; A notable feature of the deposition flux in Fig. 8(b) is that the flux near the edge toward IL-side is much higher than that near the opposite edge, which should result in the increased density of islands near to the edge of IL-side on the top terrace. Sequential formation of islands on the top terrace preferentially close to the edge makes the mound have steps with the narrower terrace width or the steeper slope on IL-side than those on SH-side.

In Fig.7, we observe the dependence of the smoothing kinetics on the side of the mound; as $T$ increases, relatively rapid smoothing occurs on both the $\mathrm{P}$ - and $\mathrm{SH}$ sides, while it is retarded at the IL-side. This is because the deposition flux is larger on the IL-side than those on the other sides (Fig. 8); The mean capture length of the deposited atoms to form islands on the IL-side is shorter than those on the other sides. It means that the effective temperature felt by the deposited atoms on the IL-side is lower than that on the other sides. As a result, the smoothing proceeds relatively slowly on the IL-side, as $T$ increases.

In summary, both (1) the inhomogeneous deposition flux over the sides of the mound due to the steering and screening effects and (2) the enhanced deposition flux near the front edge of the top terrace of the mound due to the steering effect cooperatively give rise to the asymmetric mound formation with different lengths and slopes on each side, and also accelerate the roughening of surface during deposition at grazing angle.

\section{B. The steering effects v.s. the screening effects}

As the thickness of the film become thick, in addition to the steering effects, the geometric screening effects 

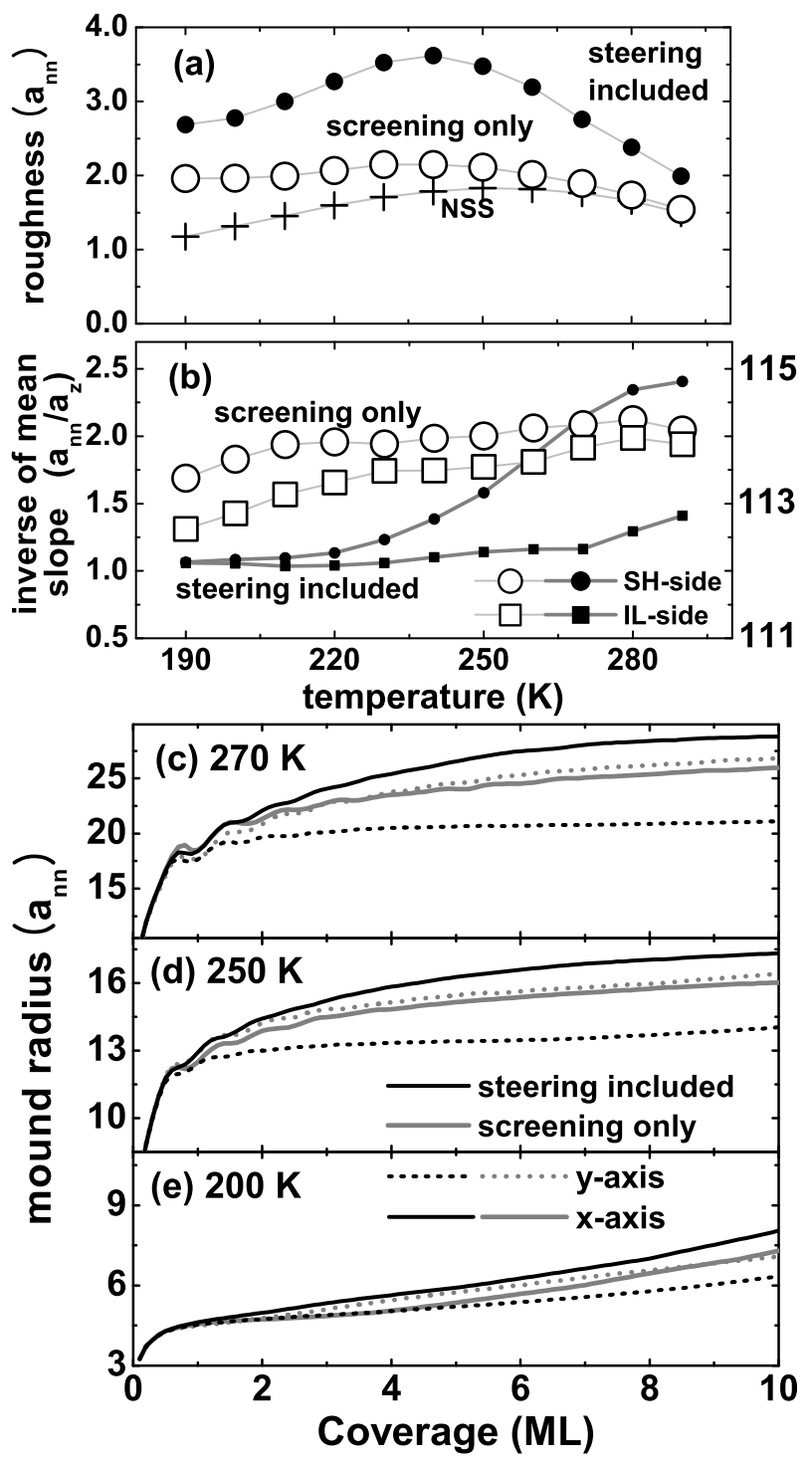

FIG. 9: Simulation results with the screening effects alone with $\theta=80^{\circ}$ and $\Theta=10 \mathrm{ML}$. As a reference, the simulation results considering both screening and steering effects are also presented. (a) Roughness as a function of temperature: open circles for deposition considering screening effects alone, + for random deposition (NSS), and closed circles for deposition considering both screening and steering effects. (b) Mean slopes for the SH-side (circle) and the IL-side (square). Open (closed) symbols for deposition including screening effects alone (both screening and steering effects). (c-e) Mound radius as a function of coverage. Gray (black) curves correspond to the deposition considering screening effects (both screening and steering effects). Solid and broken curves are radii along $\mathrm{x}$ - and $\mathrm{y}$-axis, respectively.

play an important role. To study the contribution of the individual dynamic effect to thin film growth, we carry out another set of growth simulation which takes only the geometric screening effects into account. In this simulation, the trajectory of the deposited atom is a straight line determined by the initial position and velocity of the atom.

In Fig. 9(a) shown is the roughness evolution as a function of the temperature for three different cases; (1) the case assuming no steering and screening effects (NSS) or random deposition, (2) the case taking only the geometric screening effects into account, and (3) the usual simulation taking all the dynamic effects, i.e., both steering and screening effects. At low temperature (e.g., $190 \mathrm{~K}$ ), the screening effects contribute to the roughening almost as much as the steering effects. Here, the contribution of the screening effects to the roughening is estimated as the difference between the roughness obtained with the screening effects included and that of NSS. The contribution of the steering effects is estimated similarly as the difference between the roughness with both effects considered and that with only the screening effects. The roughening due to the screening effects, however, decreases gradually as temperature increases and becomes negligible at temperatures higher than $260 \mathrm{~K}$. On the other hand, the roughness due to the steering effect keeps increasing up to $240 \mathrm{~K}$ and starts to decrease gradually at the higher temperatures. This suggests that the effects of the inhomogeneous deposition flux due to the screening effect alone relaxes through diffusion kinetics at a temperature lower than that due to the steering effects.

The origin of the difference in the roughness and the relaxation temperature between these two cases lies in their different flux distributions: In Fig. 10 shown is the flux distribution for the same mound as that in Fig. 8, but only with the screening effects taken into account. The flux near SH-side is totally depleted and about the same amount is added to IL-side. The most notable difference between two cases is found in the flux on the top terrace. The flux distribution taking both dynamic effects in Fig. 8 shows pronounced enhancement of the flux near the front edge of the top terrace, while no such enhancement of flux is found in Fig. 10. The steering effect causes vertical mass redistribution, displacing the flux on SH-side expected for the random deposition to the top terrace, accelerating the roughening of the surface. In contrast, the screening effect just redistributes the depleted flux from SH-side to IL-side in the same plane. Thus, for the relaxation of the screening effects, only the diffusions in the same plane via terrace diffusion or diffusion along the edges of mound need to be activated. For the relaxation of the steering effects, however, interlayer diffusion across the ES barrier that requires much higher activation energy than that for the in-plane diffusion should be accompanied. In fact, the bell-shape dependence of the roughness on the temperature in Fig. 9(a), shown for the case when the steering effect is also considered, is reminiscent of the growth mode where the limiting process is the diffusion over ES barrier ${ }^{13}$ Hence, the relaxation of the steering effects takes place at temperature higher than that for the relaxation of the screening effects.

In Fig. 9(b), the mean slopes for IL- and SH-sides are shown as a function of growth temperature also for the aforementioned two cases. The mean slope of the IL- side 


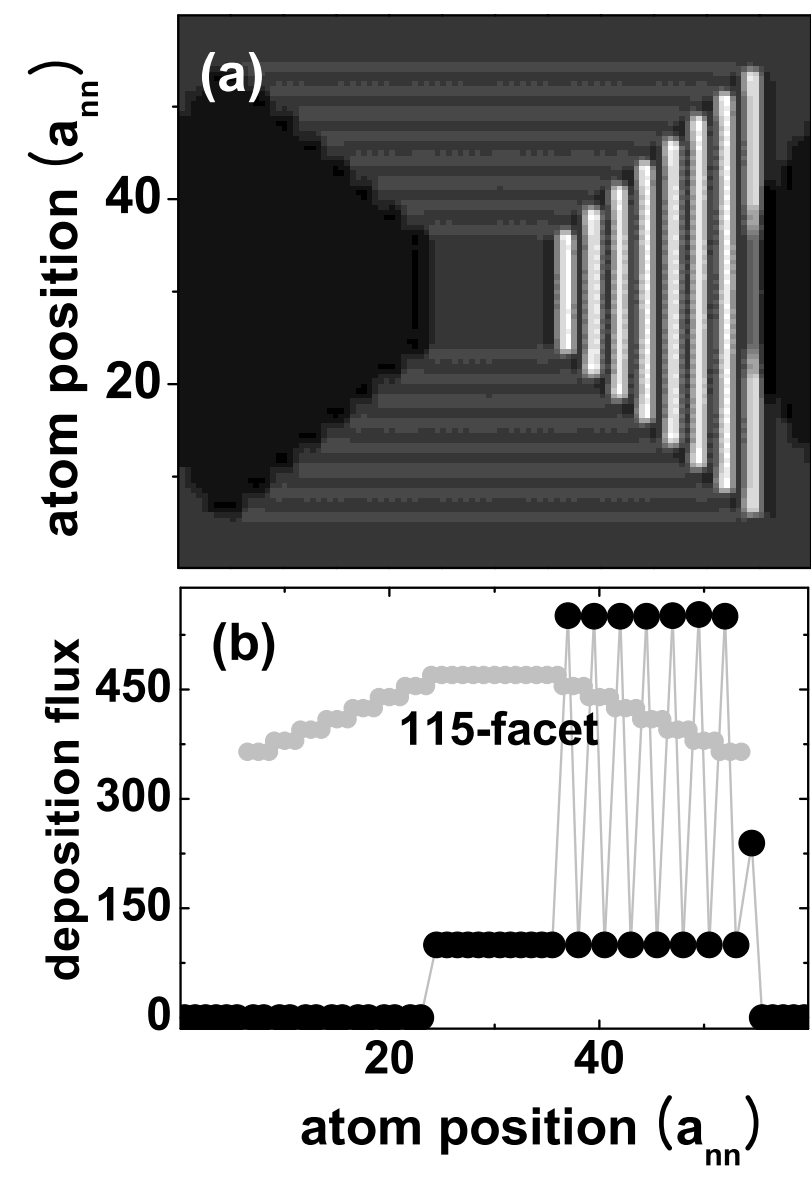

FIG. 10: Deposition flux calculated by considering only the screening effects. Atoms are deposited at grazing deposition angle of $80^{\circ}$ on an 8 -layer high mound surrounded by $(1,1,5)$ facets. (a) The local deposition flux in gray scale, where the brighter color indicates the higher local flux. (b) The local deposition flux on the line crossing the center of the mound along $\mathrm{x}$-axis is presented; the ordinate is the relative amount (\%) of deposition flux relative to the average deposition flux over the whole surface. The mound is shown with gray circles in the upper region.

is steeper than that of the SH-side over the whole temperature range for both cases. If only the screening effect is considered, the difference in their slopes dwindles as $T$ increases, and finally becomes almost negligible around $270 \mathrm{~K}$. At $250 \mathrm{~K}$, the slopes of both sides are slightly lower than that of $\{113\}$ facet. In the previous experiment depositing $40 \mathrm{ML}$ of $\mathrm{Cu}$ on $\mathrm{Cu}(001)$ at $250 \mathrm{~K}^{7}$, the slopes of the IL- and SH-side is found to be those of $\{111\}$ and $\{113\}$, respectively, in contradiction with the results of the aforementioned simulation considering the screening effects alone.

The inclusion of the steering effect makes the slopes steeper, especially on the IL-side, and the difference in slopes becomes evident as observed in the experiment. (Fig. 9(b)) Further, the mean slope of the IL-side lies in between $\{111\}$ and $\{113\}$, and that of the SH- side corresponds to that of $\{113\}$. These slopes now approach the ones observed in the previous experiment $\underline{\underline{ }}$

Regarding the mound radius, the screening effects alone make the mounds of almost symmetric shape (Figs. 9 (c)-(e)) which strikingly differs from what has been observed in the experiments ${ }^{7.14}$ This is understood by the fact that the flux blocked by the screening effect on the $\mathrm{SH}$-side results in the increase of the flux on the IL-side (Fig. 10), which looks as if the blocked flux is simply displaced to the IL side. Therefore, the reduced lateral growth speed on the SH-side is almost compensated by the increased growth speed on the IL-side. Hence, the overall shape of mound remains square symmetric, 15 if only the screening effects are taken into account in the growth. The asymmetric mounds with longer side along y-axis (Fig. 9) form as observed in the experiment, $\frac{7}{\underline{T}}$ only when the steering effect is added.

In short, in the thin film growth at grazing deposition angle, the asymmetric mound shape, the surface roughness, and the slope difference on the IL- and SH-sides of mounds result mainly due to the steering effect rather than the screening effect. (Fig. 9). Remembering that the main difference of the steering effects from the screening effects is the higher deposition flux near the front edge of the top terrace, the observed characteristics of the films grown at grazing deposition angle is largely determined by the growth characteristics of the top layer as the growth front.

\section{SUMMARY AND CONCLUSION}

We perform KMC simulation to study the thin film growth by deposition at grazing angle. We observe (1) the notable increase of the surface roughness and (2) the asymmetry in both mound shape and slopes, as compared with the thin film grown by the normal deposition. Such results are in good agreement with the previous experimental observations. We find that the aforementioned structural features of the films grown by grazing angle deposition are mainly attributed to the steering effect rather than the screening effect. Especially, the inhomogeneous deposition flux on the top terrace induced by the steering effects is the most influential factor.

We also make an additional interesting observation that the mound side is not composed of one kind of facet, even when the slope selection is attained. Instead, we find that there coexist variety of local facets and the selected mound slope observed in experiment represents only the mean slope of those. Therefore, the slope selection does not mean the facet selection.

Acknowledgments 
1 S. V. Dijken, L. C. Jorritsma, and B. Poelsema, Phys. Rev. Lett. 82,4038 (1999).

2 J. Seo, S.-M. Kwon, H.-Y. Kim, and J.-S. Kim, Phys. Rev. B 67, R121402 (2003).

${ }^{3}$ F. Montalenti, M. R. Sorensen, and A. F. Voter, Phys. Rev. Lett. 87, 126101 (2001).

4 F. Montalenti and A. F. Voter, Phys. Rev. B 64, R081401 (2001).

5 J. Yu and J. G. Amar, Phys. Rev. Lett. 89, 286103 (2002).

6 J. Seo, H.-Y. Kim, and J.-S. Kim, Phys. Rev. B 71075414 (2005).

7 S.V. Dijken, L.C. Jorritsma, and B. Poelsema, Phys. Rev. B 61, 14047 (2000).

${ }^{8}$ H. Wormeester and B. Poelsema, Phys. Rev. B 66, 165406 (2002).

9 D. E. Sanders and A. E. DePristo, Surf. Sci.254,
341(1991)

10 D.E. Sanders D.M. Halstead, and A.E. DePristo, J. Vac. Sci. Technol. A 10(4), 1986 (1992).

11 I. Furman, O. Biham, Jiang-Kai Zuo, A. K. Swan, and John F. Wendelken, Phys. Rev. B 62, R10649 (2000).

12 H. Mehl, O. Biham, I. Furman, and M. Karimi, Phys. Rev. B, 2106 (1999).

13 J.G. Amar and F. Family, Phys. Rev. B 54, 14742 (1996).

14 T.-M. Lu, D.-X. Ye, T. Karabacak, and G.-C. Wang, Mater. Res. Soc. Symp. Proc. 849, KK8.4.1 (2005).

15 The slightly longer mound length in the $y$-axis than in the $\mathrm{x}$-axis may be caused by the net mass transfer through diffusion from the IL-side to the P-side due to the large adatom density gradient resulting from the large difference in the deposition flux between the two sides. 